DOI: 10.1002/smll.(201302942)

suminus small ${ }^{100}$ Article type: Communication

\title{
Super-resolution biological microscopy using virtual imaging by a microsphere nanoscope
}

Hui Yang, Norman Moullan, Johan Auwerx, and Martin A. M. Gijs*

H. Yang, Prof. M. A. M. Gijs

Laboratory of Microsystems, École Polytechnique Fédérale de Lausanne, CH-1015 Lausanne, Switzerland

E-mail: martin.gijs@epfl.ch

Dr. N. Moullan, Prof. J. Auwerx

Laboratory of Integrative and Systems Physiology, École Polytechnique Fédérale de Lausanne, CH-1015 Lausanne, Switzerland

Keywords: fluorescence, imaging, microsphere nanoscope, super resolution

A classical microscope resolves features of around half of the wavelength of illumination $\lambda^{[1]}$.

Breaking this limit is possible in near-field scanning optical microscopy using a local

evanescent illumination spot ${ }^{[2]}$, in integral imaging three-dimensional microscope which

capture images before the diffraction effects ${ }^{[3]}$, or by applying a material of negative

refractive index, referred to as a 'superlens' ${ }^{[4-6]}$. A far-field superlens can transform scattered evanescent waves into propagating waves ${ }^{[7-8]}$, which then can be further processed by conventional optics, but its nanofabrication process is complex. Other advanced superresolution concepts are based on reducing the focal spot size, like done in confocal fluorescence microscopy ${ }^{[9]}$, modify the effective point-spread function of the excitation beam using a second laser that suppresses fluorescence emission from fluorophores located away from the center of excitation, like in stimulated emission depletion microscopy (STED) ${ }^{[10]}$, or employ photo-switchable fluorescent probes to resolve spatial differences in dense populations of molecules, as in photo-activated localization microscopy (PALM) and stochastic optical reconstruction microscopy (STORM) ${ }^{[11-12]}$. Compared to these highperformance and sophisticated techniques, a dielectric lens of plano-spherical-convex shape, known as a solid-immersion lens (SIL), remains an attractive and simple solution for 


\section{suminus small}

1 projecting information directly into the far-field ${ }^{[13-14]}$. Imaging of objects beyond the

2 diffraction limit was demonstrated with self-assembled nanoscale lenses in a SIL-type

3 implementation ${ }^{[15-17]}$, but reducing the size of a SIL to the nanoscale is challenging. Also

4 micrometer-scale spheres could convert the near-field evanescent field with high frequency

5 spatial information into a propagating mode ${ }^{[18]}$, as a 'photonic nanojet' exits from such

6 microspheres with a waist smaller than the diffraction limit ${ }^{[19-24]}$, forming the basis for their

7 superior imaging capability ${ }^{[25-28]}$. Here, we propose the use of high-refractive index $\left(n_{p}=\right.$

8 1.92) glass microspheres for facile and affordable super-resolution fluorescent imaging of

9 sub-cellular organelles and biomolecules. The microspheres are simply put on a sample that is

10 immersed in oil or water, and project the sample's near-field nano-features into the far-field,

11 generating a magnified virtual image. Using a conventional microscope objective, we image

12 control nanostructures and fluorescent nanobeads with a minimum feature size of $\sim \lambda / 7$. We

13 demonstrate the potential of the technique by resolving the structure of fluorescently stained

14 centrioles, mitochondria, chromosomes, and study the effect of doxycycline treatment on

15 mitochondrial encoded protein expression in a mouse liver cell line.

16 A schematic of several microsphere nanoscopes combined with an immersion objective is

17 illustrated in Figure 1. The transparent barium titanate glass microspheres simply self-

18 assemble on top of an object that is immersed in liquid medium with refractive index $n_{m}(1.33$

19 for experiments performed in water, or 1.52 for experiments performed in oil). Fluorescent or

20 bright-field images are obtained through a $40 \times$ water immersion objective with numerical

21 aperture $(\mathrm{NA})=0.8$, or a $63 \times$ oil immersion objective with $\mathrm{NA}=1.4$, respectively. For testing

22 the microsphere nanoscope in the fluorescent mode, a mercury vapor short arc lamp and

23 suitable filter sets for different fluorophores are mounted on the microscope, and the water

24 immersion objective is used. Figure 2 a shows the imaging of calibrated polystyrene

25 fluorescent particles with emission wavelength $\lambda_{\mathrm{p}}=680 \mathrm{~nm}$. Comparing Figure 2a(i) and

$262 \mathrm{a}(\mathrm{ii})$, demonstrates a 5.4× magnification factor when using a microsphere nanoscope with 


\section{sisuminat small}

1 diameter of $60 \mu \mathrm{m}$ for imaging a $1 \mu \mathrm{m}$-diameter particle. Subsequently, $100 \mathrm{~nm}$-diameter

2 particles are dispersed on a glass substrate, which cannot be resolved through conventional

3 fluorescent microscopy (Figure 2a(iii)). However, these particles are clearly imaged through

4 the microsphere nanoscope in Figure 2a(iv). While, without the microsphere, the waterimmersion objective has a diffraction-limited resolution of $404 \mathrm{~nm}$ at a fluorescent emission wavelength of $680 \mathrm{~nm}^{[29]}$, our fluorescence experiments demonstrate an imaging capability of an object of $\sim \lambda / 7$-size. Besides the imaging experiments in the fluorescent mode, we have also characterized in detail the technique under white light illumination, by immersing silicon

9 line nanopatterns and gold nanorod test structures in oil and imaging them through the microsphere nanoscope using an oil immersion objective. In this case we could demonstrate a

11 similar super-resolution capability, as discussed in the Supporting Information (Figure S1 $12 \mathrm{~S} 3$ ).

The image plane, on which the virtual image shows the strongest fluorescent signal is defined as the best image plane (plane ' $\mathrm{zb}$ ' in Figure $2 \mathrm{~b}$ ). To analyze the focusing behavior of the microsphere nanoscope, we use a piezo-controlled nano-positioning stage integrated with the microscope to change the microscope's focal plane precisely, and take for each plane a fluorescent image of $100 \mathrm{~nm}$ particles. On the best image plane $(\mathrm{zb}=28 \mu \mathrm{m})($ Figure $2 \mathrm{c}(\mathrm{v}, \mathrm{vi}))$, we identify a magnification factor of 5.4. When the focal plane of the microscope moves away from $\mathrm{zb}$, the $100 \mathrm{~nm}$ particles are still visible over a focal depth of several microns, though less well defined (Figure 2c(i-iv,vii-xii)). Still, such behavior is in line with the property of a 'photonic nanojet', which propagates over several optical wavelengths with a minimum beam-width smaller than the diffraction limit. To investigate the electromagnetic field distribution in the vicinity of a dielectric microsphere and provide insight into the mechanism of the nanoscope effect, we perform a finite element method (FEM) study of the 


\section{smillis \\ sibiniest small}

$12 \mathrm{~d}(\mathrm{i})$ shows the spatial distribution of the electric field when using a light source away from

2 the microsphere. The microsphere focuses the radiation into an extremely small region and

3 enhances the signal on the shadow-side of the microsphere, effectively producing the photonic nanojet. The latter possesses a waist smaller than the diffraction limit, which is at the basis of superior imaging. In Figure 2d(ii), the light source is present at the surface of the microsphere, which is the situation corresponding to our imaging experiments. By tracing the outgoing light beam trajectories from the microsphere backwards until they intersect, the position of the virtual image can be estimated. The latter is $\sim 30 \mu \mathrm{m}$ from the surface of the microsphere, while experimentally we find $\sim 28 \mu \mathrm{m}$. The simulation results clearly point out the origin of the super-resolution, which is a combination of two factors: (i) the microsphere provides local enhancement of the refractive index and a reduction of the effective wavelength, and (ii) the development of the photonic nanojet. On a fundamental level, the beam-width of the photonic nanojet directly indicates the super-resolution capability of the microsphere nanoscope. By properly selecting the optical properties of the microsphere material and its size, it is possible to optimize the setup and find conditions that lead to the minimum half-width of the nanojet, which corresponds to optimum super-resolution capability. In Figure $3, R_{\text {jet }}$ is the half-width of the nanojet and $\lambda=680 \mathrm{~nm}$ is the wavelength of the illumination. Figure $3 \mathrm{a}$ and $3 \mathrm{~b}$ show the calculated half-width of the photonic nanojet normalized by the wavelength, as function of the microsphere diameter. It is found that, when the microspheres are immersed in different media, they show different super-resolution capability. According to our simulations, a microsphere with diameter smaller than $60 \mu \mathrm{m}$ shows the ability to resolve objects with a feature size smaller than the diffraction limit, when immersed in oil (Figure 3a). However, a microsphere as big as $100 \mu \mathrm{m}$ still exhibits super-resolution, when immersed in water (Figure $3 b)$. Moreover, the influence of the microsphere refractive index $\left(n_{p}\right)$ in relation to the refractive index of the medium $\left(n_{m}\right)$ is studied. The ratio of these quantities $n_{p} / n_{m}$ is defined as the optical contrast. Figure $3 \mathrm{c}$ and $3 \mathrm{~d}$ show the maximum intensity and normalized half-width 


\section{saminas smâll}

1 of the photonic nanojet for a $60 \mu \mathrm{m}$ microsphere as a function of the optical contrast. The

2 increase of the optical contrast clearly leads to an increase in peak intensity of the photonic

3 nanojet (Figure 3c). As the optical contrast increases, the external focal point of the nanojet

4 gets closer to the microsphere, and, at a certain value of the optical contrast $\left(n_{p} / n_{m}=1.6\right)$, the

5 photonic nanojet directly emerges from the microsphere surface, which corresponds to the

6 maximum of the intensity. This condition corresponds to a refractive index $n_{p}=2.13$ for the

7 microsphere, when using water as medium. When increasing the optical contrast beyond 1.6,

8 the photonic nanojet, which "leaks out" of the microparticle, shows lower intensity.

9 Generally, the Rayleigh criterion for specifying the imaging resolution of a microscopy

system is defined as $\Delta x=1.22 \lambda /(2 \mathrm{NA})$. Considering that our method can resolve an object

11 with feature size of $100 \mathrm{~nm}$, we can say that the "effective NA" of using the $60 \mu \mathrm{m}$

12 microsphere is 4.1 . This means that the use of the $40 \times$ optical microscope objective

13 together with a $60 \mu \mathrm{m}$ microsphere nanoscope with a magnification factor of $5.4 \times$ would

14 permit a resolution that would be provided by a hypothetical (as non-existing) 216×

15 microscope objective with $\mathbf{N A}=4.1$.

The demonstrated fluorescent super-resolution microscopy in water indicates that our technique could be extremely interesting for life science applications. We perform imaging experiments on several different organelles in the alpha mouse liver 12 (AML12) cell line, like centrosomes, mitochondria, and chromosomes. We first image centrioles, which form the core of the centrosome and are primarily composed of $\gamma$-tubulin proteins. In eukaryotic cells, centriole cylinder-like structures are built of nine triplet microtubules and have approximate diameters of $150-200 \mathrm{~nm}$ and lengths of $400-450 \mathrm{~nm}^{[30-31]}$. Each centrosome comprises two centrioles, of which, the older one, frequently referred to as the mother, is perpendicular to the other ${ }^{[32]}$. Conventional optical microscopy can reveal the presence of centrioles but not their sub-structure, as shown in Figure 4(a,c). Here, an anti- $\gamma$-tubulin antibody $(\mathrm{Ab})$ and 


\section{sisuinitut Small}

1 fluorescently label secondary Ab is used to stain $\gamma$-tubulin (in red), while Hoechst 33342 is

2 used to stain the nucleus (in blue). When a microsphere nanoscope is placed on top of the

3 centriole(s), we can detect $\gamma$-tubulin as a dot at the center and the surrounding ring of the

4 mother centriole (Figure $4 \mathrm{~b}$ ), or we can image the link between the mother and the daughter centriole (Figure 4d). We also perform imaging experiments on mitochondria and

6 chromosomes, the former being stained by MitoTracker® probe and the latter by 4', 6-

7 diamidin-2-phenylindol (DAPI). Figure 4(e,g) and 3(f,h) show the fluorescent images of

8 mitochondria and chromosomes, obtained using conventional optical microscopy and a

9 microsphere nanoscope, respectively. The latter can better resolve their complex shapes than conventional microscopy indeed. It should be noted that all the fluorescent images are taken

11 using a commonly used $40 \times$ water immersion objective with $\mathrm{NA}=0.8$, which is able to

12 monitor a sample over a large field-of-view (FOV) $(225 \mu \mathrm{m} \times 168 \mu \mathrm{m})$ for observing

13 simultaneously many microsphere nanoscopes. Therefore, not only we can beat the diffraction

14 limit, but we are able to image a population of nanometer-sized structures, which is key to any

15 biological study where coverage of a large area is required to yield representative images with

16 statistical relevance (see for example in Figure 5).

17 The subcellular locations of proteins are closely related to their function and constitute an

18 essential aspect for understanding the complex machinery of living cells. However, due to the

19 diffraction limit, conventional optical microscopy cannot provide much information on the 20 localization of the proteins. Here, we study the imaging with the microsphere nanoscope of 21 proteins in AML12 cells, and more specifically of the mitochondrial encoded cytochrome c oxidase I (MTCO1), a protein that is part of respiratory complex IV. It has been recently

23 shown that antibiotics targeting the mitochondrial translation machinery will cause

24 mitonuclear protein imbalance, as proteins encoded by nuclear and mitochondrial DNA need

25 to be stochiometrically matched in the complexes and supercomplexes of the respiratory chain 


\section{smontlis \\ Suburited to Small}

$1{ }^{[33]}$. An expression difference between mitochondrial (MTCO1) and nuclear encoded proteins,

2 such as succinate dehydrogenase complex, subunit A (SDHA), will activate a specific

3 mitochondrial unfolded protein response (UPR $\left.{ }^{\mathrm{mt}}\right)$, which is a reparative pathway that reduces

4 the amount of unfolded proteins and restores mitochondrial proteostasis. The activation of this

5 pathway hence protects the mitochondria and may contribute to longevity. We hence treated

6 AML12 cells with doxycycline during 48 hours at a concentration of $30 \mu \mathrm{g} / \mathrm{mL}$ or with the

7 vehicle dimethyl sulfoxide (DMSO) as a control. Figure 6 shows that the level of MTCO1

8 decreases dramatically with the treatment of doxycycline. With conventional microscopy, it is

9 extremely difficult to observe the localization of proteins in the cells, and it is hard to identify

10 differences in the distribution of MTCO1 upon doxycycline treatment. When a microsphere

11 nanoscope is used, the state of localization of MTCO1 protein is clearly observed. Figure 6(a)

12 shows important MTCO1 protein expression, while this decreases robustly upon the

13 doxycycline treatment. A western blot showing the corresponding differences in MTCO1

14 versus SDHA expression is shown in Figure 6(b) and the ratio between the expression levels

15 of the two proteins is calculated in Figure 6(c). The imbalance of MTCO1 and SDHA protein

16 with the doxycycline treatment is shown in detail in the Supporting Information (Figure S5).

17 It should be noticed that the integrated fluorescent signal intensity of a virtual image is never

18 larger than the integrated intensity emitted by the (non-magnified) sample. We believe this is

19 due to two reasons: first, when the objective focal plane coincides with the virtual image

20 plane, it may be not optimally focused on the fluorophores, hence, the excitation of the

21 fluorescence is less efficient; second, light absorbance in the dielectric medium (glass

22 microsphere) cannot be neglected.

23 In conclusion, we achieve super-resolution imaging of nanostructures with sub-diffraction

24 feature sizes by using a microsphere nanoscope in combination with a conventional

25 immersion objective. The fluorescence experiments performed in water show that an object 


\section{sibuiniet s s Small}

1 with a size as small as $\sim \lambda / 7$, i.e. one fourth of the diffraction limit, can be quantitatively

2 determined, while a $5.4 \times$ magnification of the image is obtained. Subsequently, we image

3 biological samples and demonstrate that the shape of subcellular structures like centrioles,

4 mitochondria and chromosomes can be clearly resolved through the microsphere nanoscope.

5 The imaging method is also used to identify the expression of the specific mitochondrial

6 membrane protein MTCO1. We think that in future, due to the straightforwardness of this

7 approach, the microsphere nanoscope will be a robust and versatile tool that can be used to

8 image with a conventional microscope a variety of biological objects such as viruses, nucleic

9 acids, and functional organelles in living cells, and this at virtually no extra cost.

10

11

\section{Experimental Section}

Nano-scale test structures, microspheres, and microscopy: The calibrated polystyrene

fluorescent particles were obtained from Invitrogen (Basel, Switzerland). The barium titanate glass microspheres were obtained from Cospheric (Santa Barbara, CA, USA). The optical microscopy images were obtained by using a Zeiss Axioplan microscope mounted with a AxioCam MRm camera (Carl Zeiss GmbH, Oberkochen, Germany). Phosphate buffered saline (PBS, pH 7.4) and de-ionized water were used as liquid medium for the fluorescence experiments with the cells and particles, respectively.

Numerical simulation: The numerical study of the microsphere nanoscope effect is carried out by FEM in COMSOL Multiphysics software. A scalar equation is used to study transverse electric waves propagating through the microsphere and the surrounding media. An emission wavelength of $680 \mathrm{~nm}$ is used as the wavelength of the light source. After meshing, the element size is $55 \mathrm{~nm}$, i.e. $\sim$ one twelfth of the wavelength, which is sufficiently small to obtain a precise solution.

Cell experiments: The mouse hepatocyte cell line AML12 was obtained from ATCC (Manassas, VA, USA). Cells were grown at $37^{\circ} \mathrm{C}$ in a humidified atmosphere of $5 \%$ 


\section{smolles \\ sistinate $s$ mall}

$1 \mathrm{CO}_{2} / 95 \%$ air in Dulbecco's Modified Eagle Medium / Nutrient Mixture F-12 (DMEM/F-12)

2 and supplemented with $0.005 \mathrm{mg} / \mathrm{mL}$ insulin, $0.005 \mathrm{mg} / \mathrm{mL}$ transferrin, $5 \mathrm{ng} / \mathrm{mL}$ selenium, 40

$3 \mathrm{ng} / \mathrm{mL}$ dexamethasone, and 10\% fetal bovine serum without antibiotics. Rabbit polyclonal antibody to $\gamma$-tubulin (anti- $\gamma$-tubulin $\mathrm{Ab}$ ) and goat polyclonal secondary $\mathrm{Ab}$ to rabbit $\operatorname{IgG}$ (DyLight ${ }^{\circledR}$ 649-conjugated) obtained from Abcam (Cambridge, UK) were used as the centriole markers. Hoechst 33342, MitoTracker ${ }^{\circledR}$ Deep Red FM probe and DAPI were obtained from Invitrogen (Basel, Switzerland), and used to stain DNA, mitochondria and chromosomes, respectively. The details on the staining of different subcellular structures are shown in the Supporting Information. Rabbit anti-COX1/MTCO1 polyclonal Ab (Cy5conjugated) and rabbit anti-SDHA polyclonal Ab (FITC-conjugated) obtained from Bioss (Woburn, MA, USA) were used as MTCO1 and SDHA marker, respectively. Western blotting was performed to check the protein level in the cells with or without doxycycline treatment. The experimental procedure is presented in detail in the Supporting Information.

\section{Acknowledgements}

The authors would like to thank L. Baldi-Unser, C. Vandevyver, H. C. Tekin, A. T. Ciftlik, A. Seitz, and Y. Zhang for discussions, and C. Huang for helping with the cell experiments. Funding of this work was provided by the EPFL, the Swiss National Science Foundation (Grant 200020-121558, 200020-140328, 31003A-124713 and CRSII3136201), the EU Ideas program (ERC-2008-AdG-23118 and ERC-2012-AdG-320404), and the Velux Stiftung. J. A. is the Nestlé Chair in Energy Metabolism.

Received: ((will be filled in by the editorial staff))

Revised: ((will be filled in by the editorial staff)) Published online: ((will be filled in by the editorial staff))

[1] L. Novotny, B. Hecht, Principles of nano-optics, Cambridge Univ. Press, New York,

\section{USA 2006.}


1 [3] R. Rajasekharan, T. D. Wilkinson, P. J. W. Hands, Q. Dai, Nano Lett. 2011, 11, 27702773.

3 [4] J. B. Pendry, Phys. Rev. Lett. 2000, 85, 3966-3969.

4 [5] N. Fang, H. Lee, C. Sun, X. Zhang, Science 2005, 308, 534-537.

5 [6] X. Zhang, Z. Liu, Nat. Mater. 2008, 7, 435-441.

6 [7] I. I. Smolyaninov, Y.-J. Hung, C. C. Davis, Science 2007, 315, 1699-1701.

7 [8] J. Rho, Z. Ye, Y. Xiong, X. Yin, Z. Liu, H. Choi, G. Bartal, X. Zhang, Nat. Commun. $8 \quad 2010,1: 143$, doi: $10.1038 /$ ncomms 1148.

9 [9] J. B. Pawley, Handbook of Biological Confocal Microscopy, Springer, New York, 10 USA 2006.

11 [10] S. W. Hell, J. Wichmann, Opt. Lett. 1994, 19, 780-782.

12 [11] E. Betzig, G. H. Patterson, R. Sougrat, O. W. Lindwasser, S. Olenych, J. S. Bonifacino,

M. W. Davidson, J. Lippincott-Schwartz, H. F. Hess, Science 2006, 313, 1642-1645.

14 [12] M. J. Rust, M. Bates, X. Zhuang, Nat. Methods 2006, 3, 793-795.

[13] S. M. Mansfield, G. S. Kino, Appl. Phys. Lett. 1990, 57, 2615-2616.

[14] D. Kang, C. Pang, S. M. Kim, H. S. Cho, H. S. Um, Y. W. Choi, K. Y. Suh, $A d v$.

Mater. 2012, 24, 1709-1715.

[15] K. S. Kim, SPIE Newsroom 2010, doi: 10.1117/2.1201004.002883.

19 [16] J. Y. Lee, B. H. Hong, W. Y. Kim, S. K. Min, Y. Kim, M. V. Jouravlev, R. Bose, K. S. 20 Kim, I.-C. Hwang, L. J. Kaufman, C. W. Wong, P. Kim, K. S. Kim, Nature 2009, 460, 49821501.

22 [17] D. R. Mason, M. V. Jouravlev, K. S. Kim, Opt. Lett. 2010, 35, 2007-2009.

23 [18] Z. B. Wang, N. Joseph, L. Li, B. S. Luk’yanchuk, J. Mech. Eng. Sci. 2010, 224, 1113241127.

25 [19] H. Rigneault, J. Capoulade, J. Dintinger, J. Wenger, N. Bonod, E. Popov, T. W.

26 Ebbesen, P.-F. Lenne, Phys. Rev. Lett. 2005, 95, 117401. 
1 [20] A. Heifetz, S.-C. Kong, A. V. Sahakian, A. Taflove, V. Backman, J. Comput. Theor.

2 Nanosci. 2009, 6, 1979-1992.

3 [21] M.-S. Kim, T. Scharf, S. Mühlig, C. Rockstuhl, H. P.Herzig, Opt. Express 2011, 19,

$4 \quad 10206-10220$.

5 [22] P. Ferrand, J. Wenger, A. Devilez, M. Pianta, B. Stout, N. Bonod, E. Popov, H.

$6 \quad$ Rigneault, Opt. Express 2008, 16, 6930-6940.

7 [23] H. Yang, M. A. M. Gijs, Anal. Chem. 2013, 85, 2064-2071.

8 [24] A. V. Itagi, W. A. Challener, J. Opt. Soc. Am. A 2005, 22, 2847-2858.

9 [25] Z. Wang, W. Guo, L. Li, B. Luk'yanchuk, A. Khan, Z. Liu, Z. Chen, M. Hong, Nat. 10 Commun. 2011, 2, doi: 10.1038/ncommns1211.

11 [26] Z. Chen, A. Taflove, Opt. Express 2004, 12, 1214-1220.

12 [27] A. Darafsheh, G. F. Walsh, L. Dal Negro, V. N. Astratov, Appl. Phys. Lett. 2012, 101, 13141128.

14 [28] Y. E. Geints, E. K. Panina, A. A. Zemlyanov, Opt. Commun. 2010, 283, 4775-4781.

15 [29] D. J. Goldstein, J. Microsc. 1992, 166, 185-197.

16 [30] E. A. Nigg, J. W. Raff, Cell 2009, 139, 663-678.

17 [31] J. L. Badano, T. M. Teslovich, N. Katsanis, Nature Rev. Genet. 2005, 6, 194-205.

18 [32] M. Bornens, Science 2012, 335, 422-426.

19 [33] R. H. Houtkooper, L. Mouchiroud, D. Ryu, N. Moullan, E. Katsyuba, G. Knott, R. W.

20 Williams, J. Auwerx, Nature 2013, 497, 451-459. 


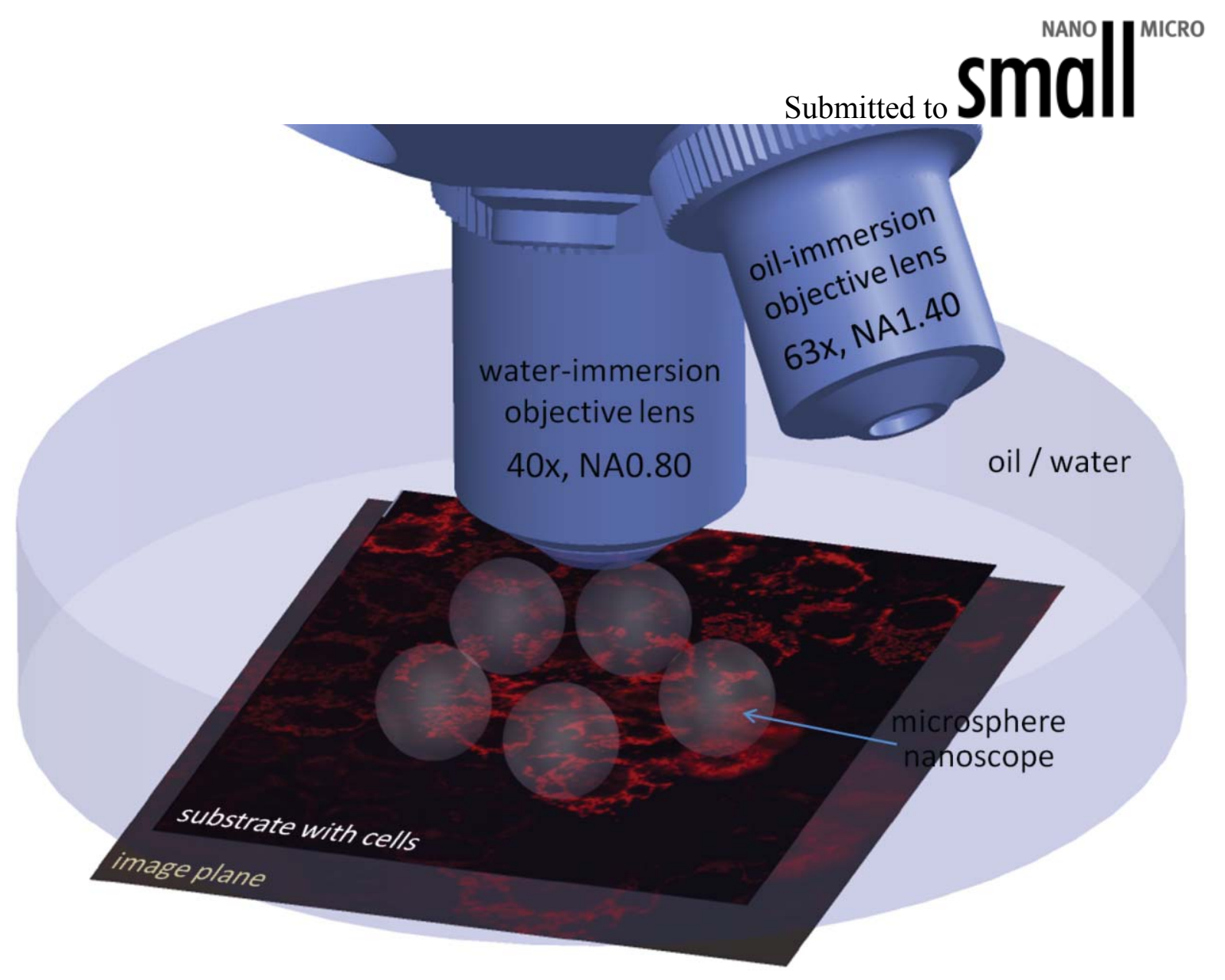

2 Figure 1. Schematic of the microsphere nanoscope. Transparent glass microspheres are 3 located on top of cells and project near-field optical information into the far-field, generating 4 magnified virtual images that are observable by a classical microscope objective. The 5 microspheres can resolve sub-diffraction features and act as superlenses in water or oil owing 6 to their high refractive index. 
(a)

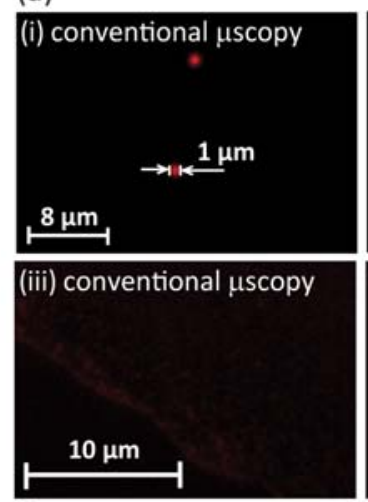

(b)
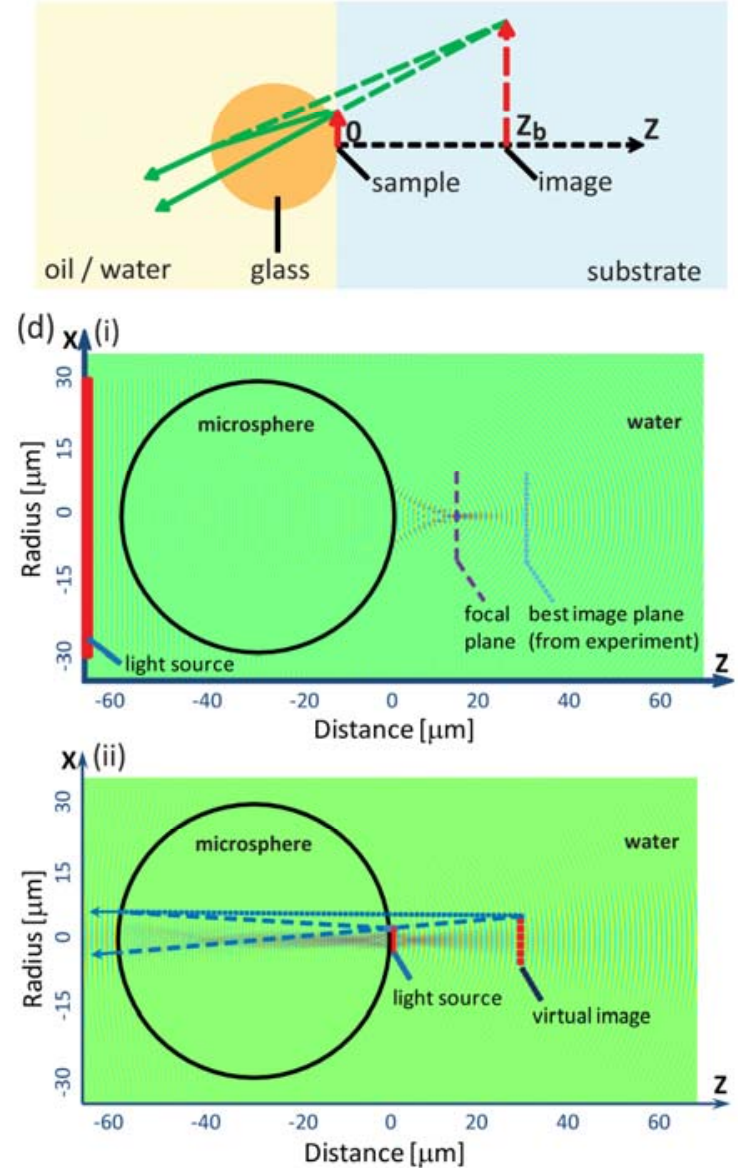

(c)

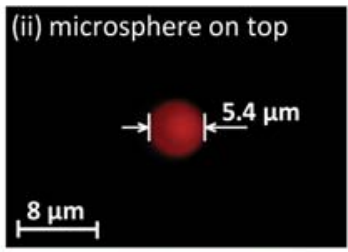

(iv) microsphere on top
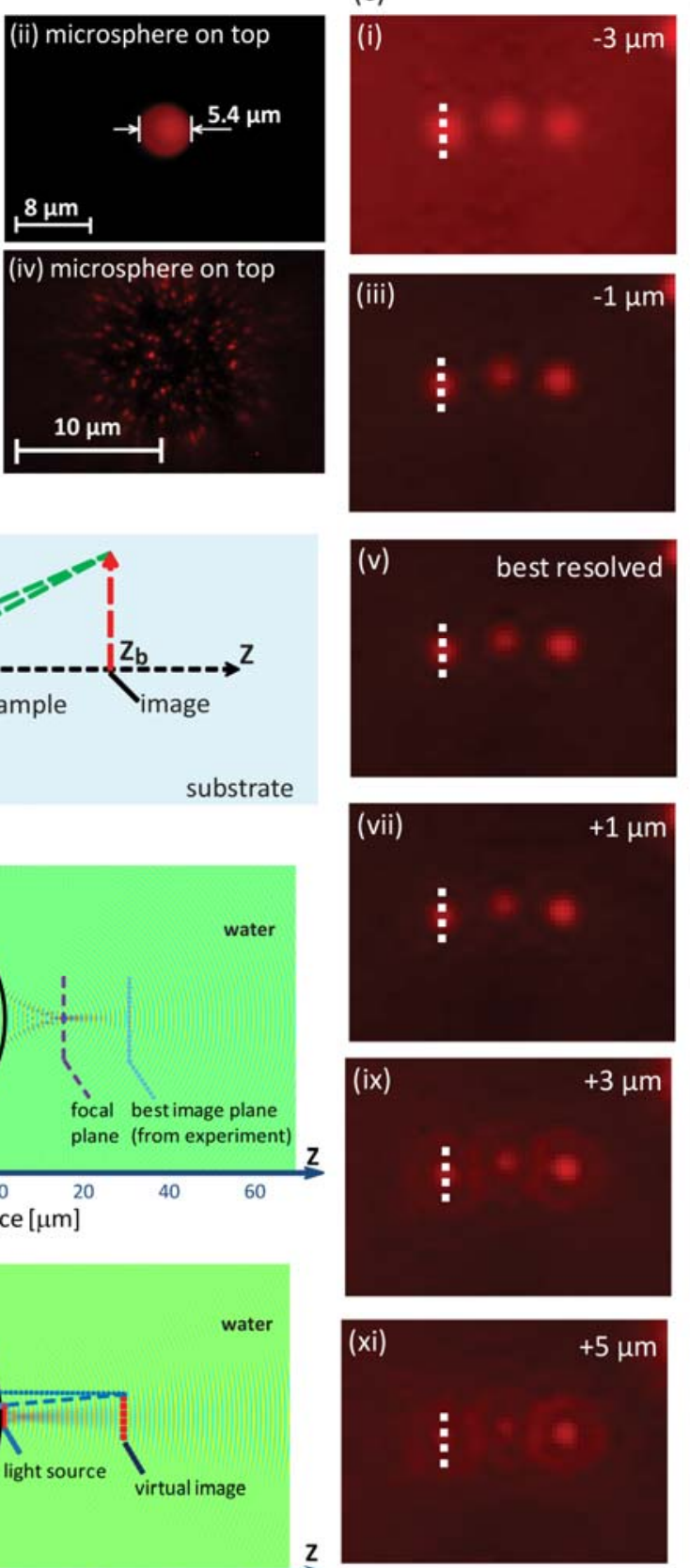
small

Submitted to
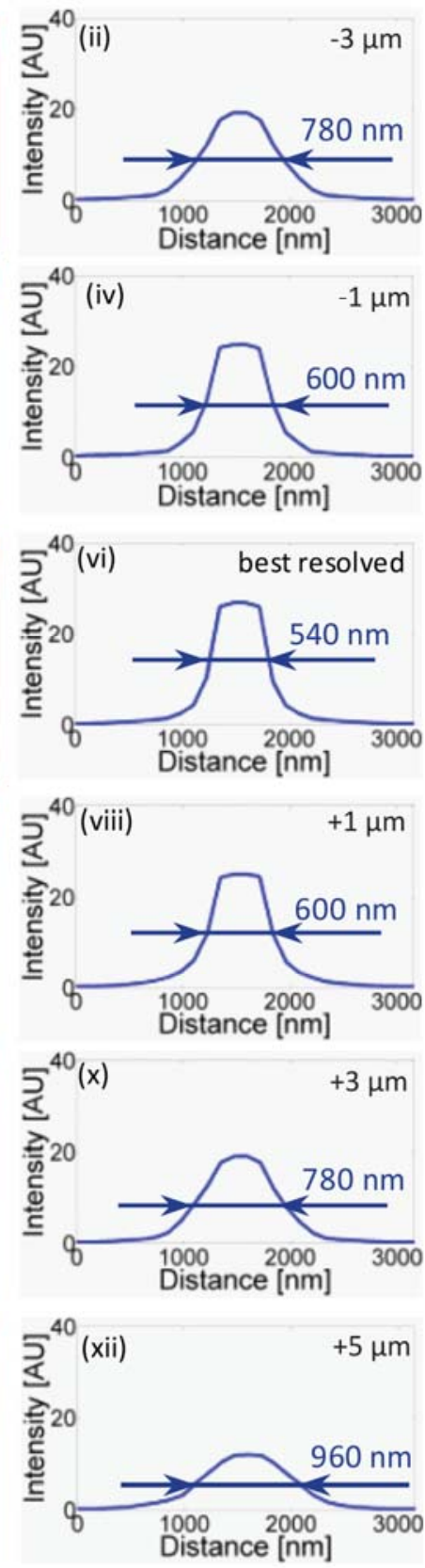

Figure 2. Super-resolution fluorescent imaging by a microsphere nanoscope. (a) Comparison of conventional microscopy and use of a microsphere for fluorescent imaging of micro-/nanoparticles: $1 \mu \mathrm{m}$ fluorescent particles imaged by (i) conventional microscopy and (ii) using a microsphere showing a magnification factor of 5.4; $100 \mathrm{~nm}$ fluorescent particles imaged by (iii) conventional microscopy, showing that these nanoparticles cannot be resolved, and (iv) using a microsphere. (b) Diagram of the virtual image formation by a microsphere nanoscope. The best image plane is defined as $\mathrm{z}=\mathrm{zb}$. (c) Virtual images and intensity profiles of $100 \mathrm{~nm}$ fluorescent particles projected through a $60 \mu \mathrm{m}$ microsphere in water on image planes at varying distances along the z-direction, (v) \& (vi) show the best resolved image at the plane with $\mathrm{z}=\mathrm{zb}=28 \mu \mathrm{m}$; ( $\mathrm{i}$ - iv, vii - xii) are the virtual images and intensity profiles at $\mathrm{z}-\mathrm{Zb}=-3$, $1,+1,+3,+5 \mu \mathrm{m}$, respectively. (d) Comsol simulations of the light propagation through a microsphere: (i) light incident from the left results in a photonic nanojet, and (ii) a light source placed on the surface of the microsphere results in a virtual image. 

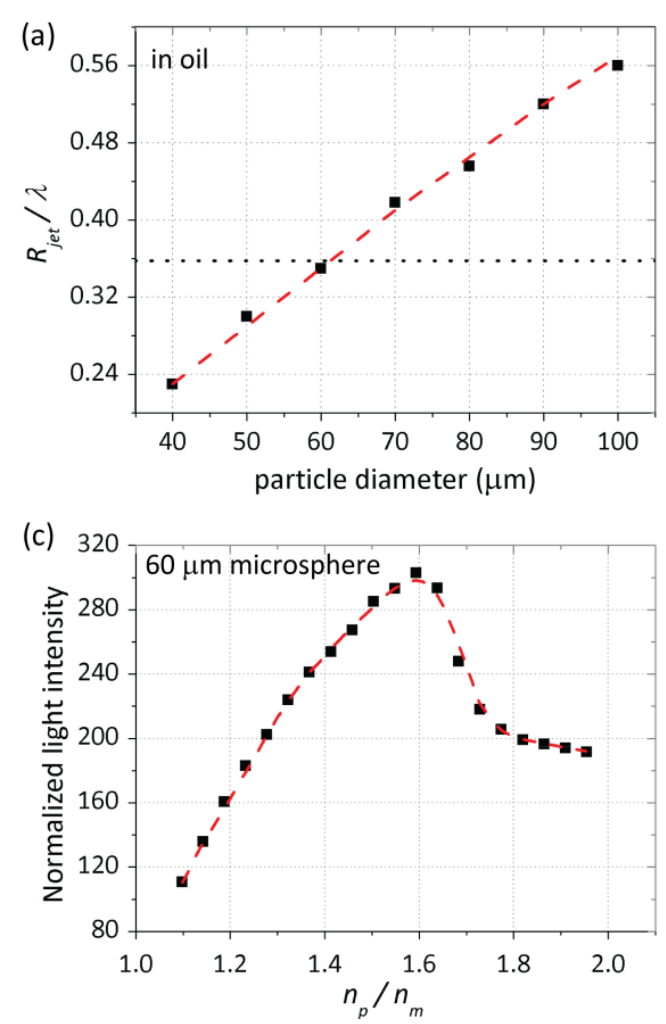

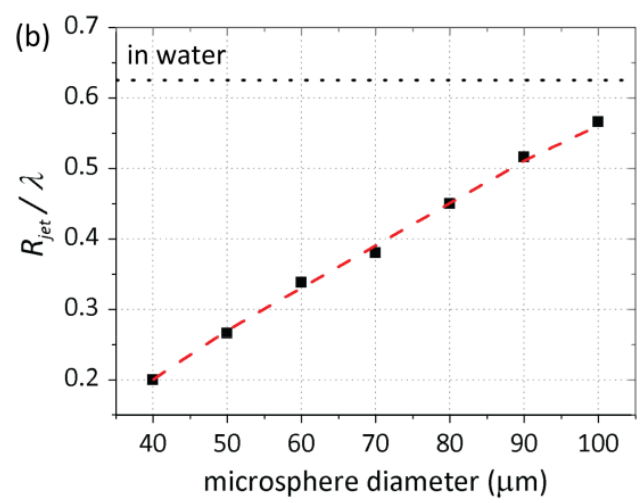

(d)

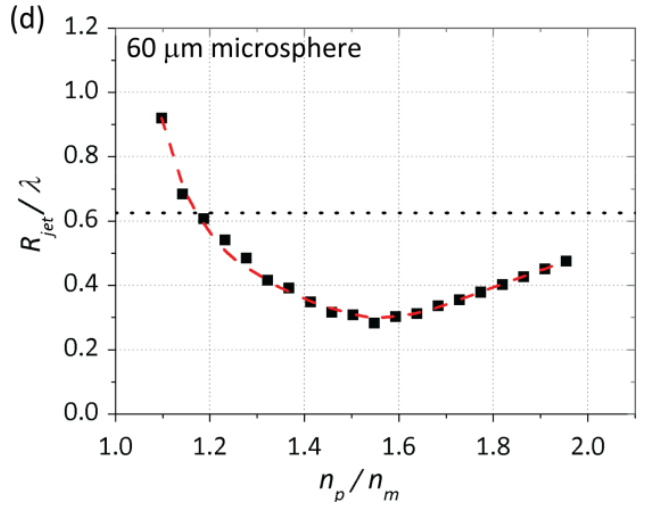

Figure 3. FEM simulation results of the light intensity showing $(a, b)$ the half-width of the photonic nanojet normalized by the wavelength as function of the microsphere diameter, the latter being immersed in oil or water, respectively. The squares are values obtained from the simulation and the dashed lines are guides to the eye. The dotted lines corresponds to the diffraction limit of an oil $(\mathrm{NA}=1.40)$ or a water $(\mathrm{NA}=0.80)$ immersion objective. (c)

8 Maximum intensity at the focal plane and (d) normalized half-width of the photonic nanojet 9 as function of the optical contrast $n_{p} / n_{m}$, when using a $60 \mu \mathrm{m}$ microsphere. In (d), the dotted 10 line corresponds to the diffraction limit of a water immersion objective $(\mathrm{NA}=0.80)$. 
canius small ${ }^{2}$
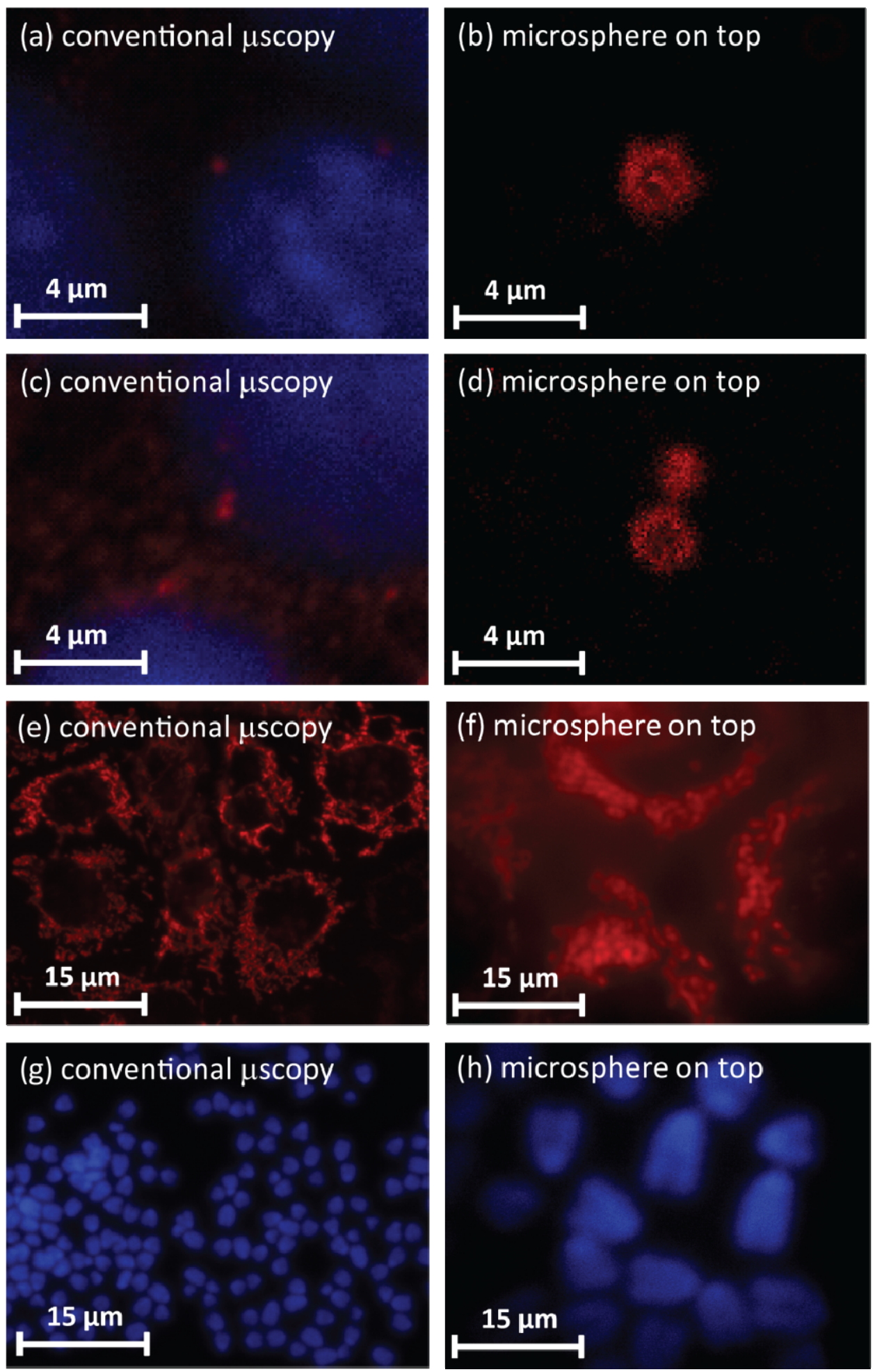

Figure 4. Fluorescent microscopy of biological objects. Comparison of conventional fluorescent microscopy (left column) and the use of a microsphere nanoscope (right column) to image different sub-cellular structures in AML12 cells. (a) $\gamma$-tubulin on a single centriole (in red) is detected as a faint spot, but in (b) the dot-in-ring structure is clearly resolved. Staining is by anti- $\gamma$-tubulin $\mathrm{Ab}$ and Dylight ${ }^{\circledR} 649$ conjugated secondary Ab; Hoechst 33342 is used to stain the nucleus (in blue). (c,d) $\gamma$-tubulin detected on a mother and daughter 8 centriole. $(\mathrm{e}, \mathrm{f})$ Images of mitochondria, stained by MitoTracker ${ }^{\circledR}$ probe. $(\mathrm{g}, \mathrm{h})$ Images of 9 chromosomes, stained by DAPI. 


\section{Submitted to}
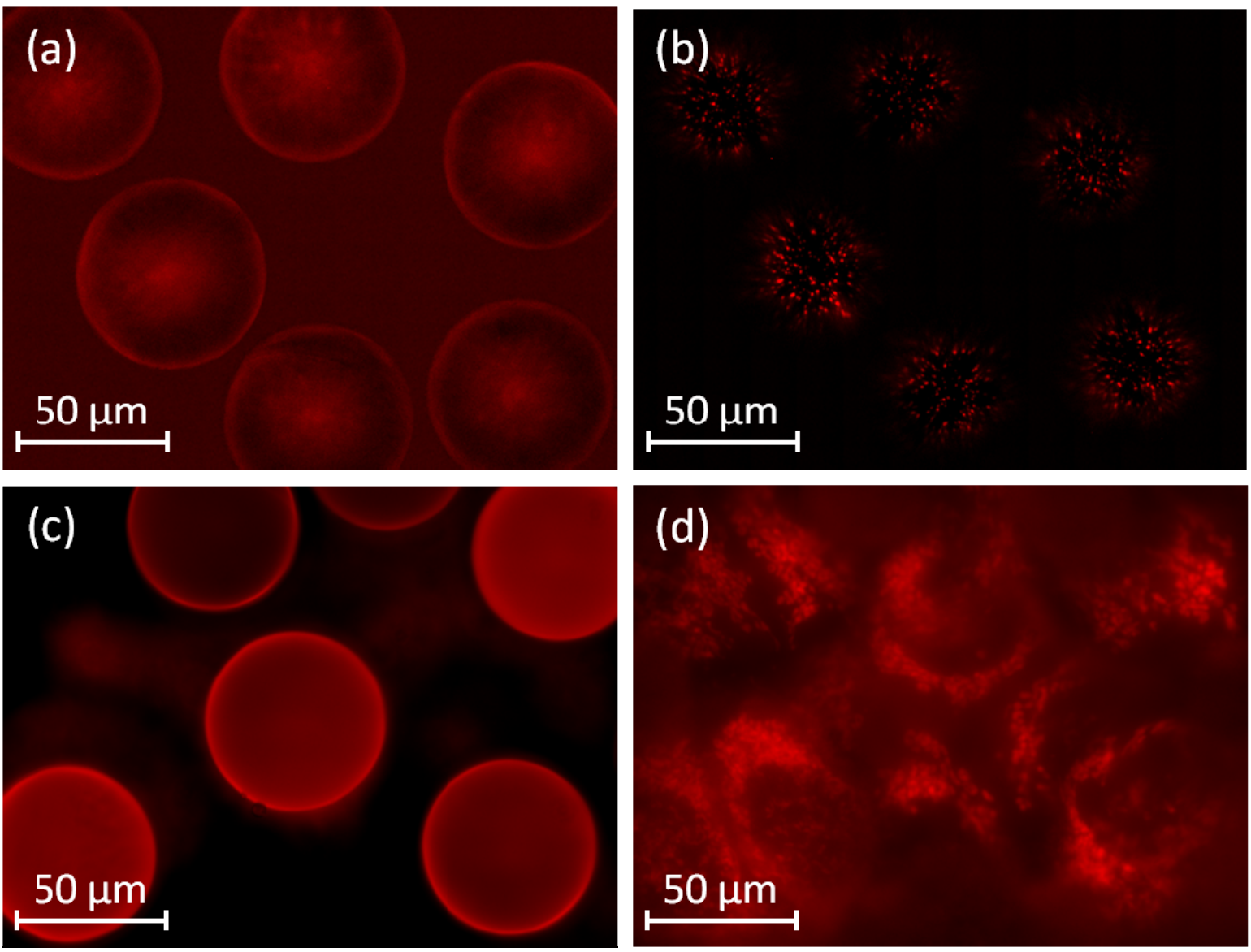

2 Figure 5. Multiple microsphere nanoscopes within the FOV of the microscope objective. 3 Fluorescent pictures showing multiple microsphere nanoscopes within the FOV of a $40 \times$

4 water immersion objective $(\mathrm{NA}=0.8)$ and placed (a) on $100 \mathrm{~nm}$ diameter nanoparticles that 5 are distributed on a glass substrate, and (c) on AML12 cells with mitochondria stain. The two 6 graphs are focused on the plane where the section of the microspheres is largest. (b,d) Virtual 7 images of simultaneous detection of multiple areas with (b) $100 \mathrm{~nm}$ nanoparticles and (d)

8 mitochondria, obtained by focusing the objective at the plane $\mathrm{z}=\mathrm{zb}$. 
(a)

\section{sumensmall}

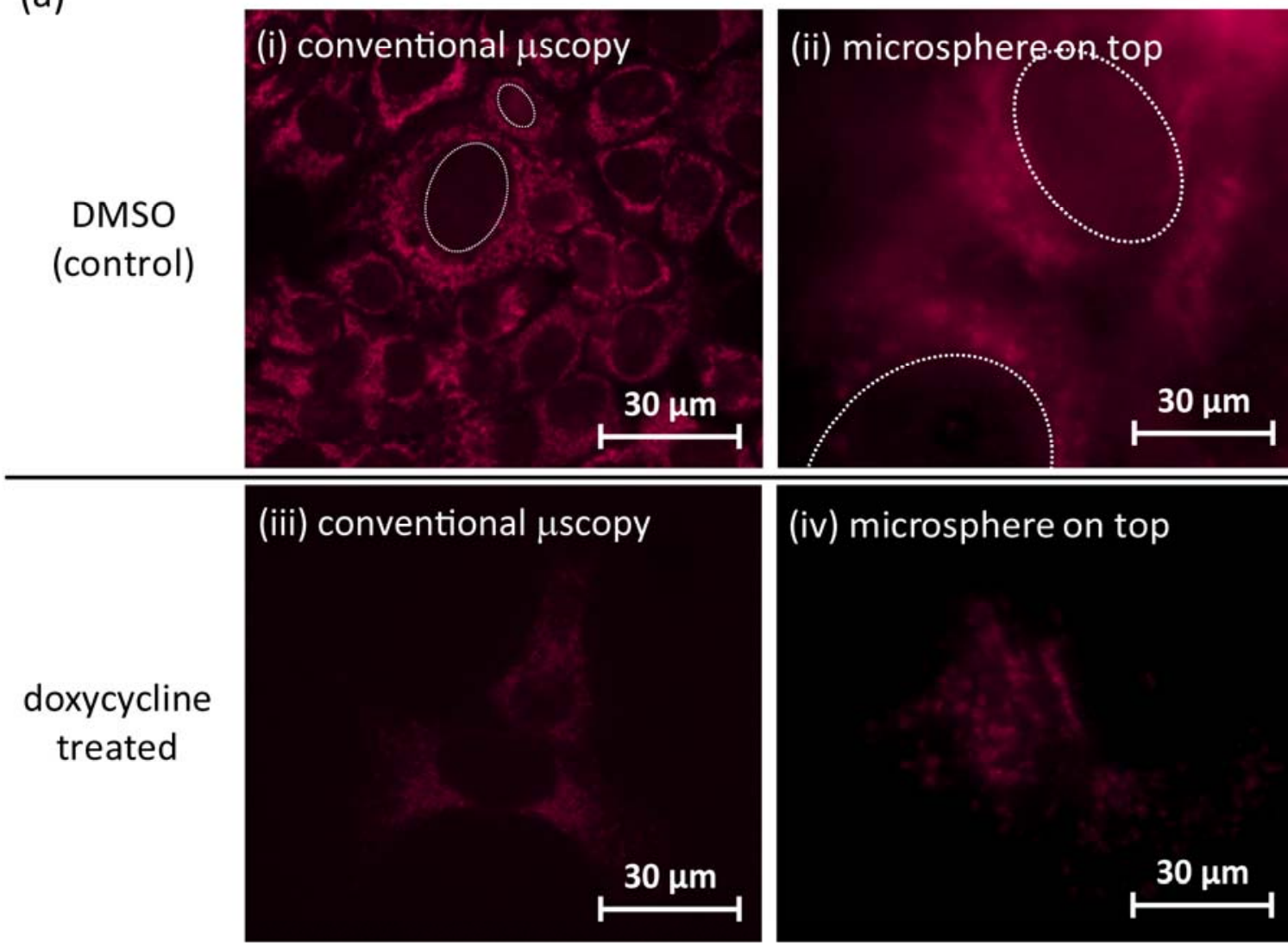

(b) dox. ( $\mu \mathrm{g} / \mathrm{mL})$

0

30

\section{MTCO1} (complex IV)

\section{SDHA (complex II)}

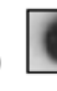

Tubulin (c)

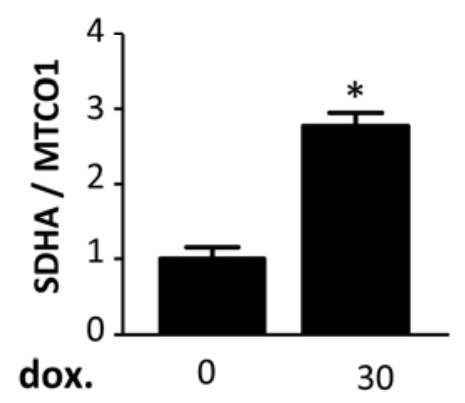

Figure 6. Study the effect of doxycycline treatment on mitochondrial encoded protein (MTCO1) expression. (a) Fluorescent images showing the distribution of MTCO1 proteins using conventional microscopy (left column) and the microsphere nanoscope (right column). Compared to the control experiments with DMSO shown in (i,ii), doxycycline treatment diminishes MTCO1 expression, as shown in (iii,iv). The black ellipsoidal areas in (i-iii) are nuclei (some have been indicated by dotted lines), in which there is no MTCO1 expression; the latter only exists in the cytoplasm. (b) Western-blot analysis of the expression of nuclear encoded SDHA and mitochondrial encoded MTCO1, using tubulin as control. A 48 hours treatment with doxycycline $(30 \mu \mathrm{g} / \mathrm{mL})$ results in a decrease of MTCO1, but not of SDHA expression. (c) The ratio of nuclear and mitochondrial encoded protein expression, obtained from the intensity of the bands in the Western-blot with and without doxycycline treatment, is statistically significant (the ' $*$ ' mark corresponds to a p-value of 0.05 ). 